\title{
Vital signs and their cross-correlation in sepsis and NEC: a study of 1,065 very-low-birth-weight infants in two NICUs
}

\author{
Karen D. Fairchild', Douglas E. Lake², John Kattwinkel', J. Randall Moorman², David A. Bateman², Philip G. Grieve3, \\ Joseph R. Isler ${ }^{3}$ and Rakesh Sahni ${ }^{3}$
}

BACKGROUND: Subtle changes in vital signs and their interactions occur in preterm infants prior to overt deterioration from late-onset septicemia (LOS) or necrotizing enterocolitis (NEC). Optimizing predictive algorithms may lead to earlier treatment.

METHODS: For 1,065 very-low-birth-weight (VLBW) infants in two neonatal intensive care units (NICUs), mean, SD, and cross-correlation of respiratory rate, heart rate (HR), and oxygen saturation $\left(\mathrm{SpO}_{2}\right)$ were analyzed hourly (131 infant-years' data). Cross-correlation (cotrending) between two vital signs was measured allowing a lag of $\pm 30 \mathrm{~s}$. Cases of LOS and NEC were identified retrospectively $(n=186)$ and vital sign models were evaluated for ability to predict illness diagnosed in the ensuing $24 \mathrm{~h}$.

RESULTS: The best single illness predictor within and between institutions was cross-correlation of $\mathrm{HR}-\mathrm{SpO}_{2}$. The best combined model (mean $\mathrm{SpO}_{2}, \mathrm{SDHR}$, and cross-correlation of $\mathrm{HR}-\mathrm{SpO}_{2}$ ) trained at one site with $\mathrm{ROC}$ area 0.695 had external $\mathrm{ROC}$ area of 0.754 at the other site, and provided additive value to an established HR characteristics index for illness prediction (Net Reclassification Improvement: 0.205; 95\% confidence interval (CI): 0.113, 0.328).

CONCLUSION: Despite minor inter-institutional differences in vital sign patterns of $V L B W$ infants, cross-correlation of $H R-\mathrm{SpO}_{2}$ and a 3-variable vital sign model performed well at both centers for preclinical detection of sepsis or NEC.

A nalysis of changes in vital sign patterns in hospitalized patients can yield important information about impending clinical deterioration and might alert clinicians before they would otherwise recognize signs of illness (1-3). We previously developed a system for analyzing heart rate characteristics in infants in the neonatal intensive care unit (NICU) that identifies decreased heart rate variability and decelerations that may occur prior to diagnosis of sepsis (4-6). Displaying a heart rate characteristics score to clinicians lowered sepsisassociated mortality $40 \%$ in a large randomized clinical trial of very-low-birth-weight (VLBW) infants (7). While changes in heart rate patterns can provide some information about cardiovascular stability and autonomic nervous system activation and dysfunction, changes in other vital signs that occur during a systemic inflammatory response can be exploited for predictive monitoring $(8,9)$.

Acute illness in preterm infants is often associated with increased frequency or severity of central apnea associated with bradycardia and oxygen desaturation ("ABD" events). We recently used an automated algorithm that analyzes waveform and vital sign data from NICU bedside monitors to show that $\mathrm{ABD}$ events and periodic breathing increase in the day prior to diagnosis in some infants with septicemia or necrotizing enterocolitis (NEC) $(10,11)$. Waveform data are generally sampled at high frequency by standard ICU monitors (in our units, chest impedance at $60 \mathrm{~Hz}$ and 3 leads of ECG at $240 \mathrm{~Hz}$ each), and therefore analysis of central apnea requires very large data storage and processing capabilities not available at most centers. In the current study, we sought to develop simpler methods for analyzing vital sign values and their interactions to predict acute illness. We focused on vital signs collected every $2 \mathrm{~s}(0.5 \mathrm{~Hz})$ from bedside monitors: heart rate (HR), respiratory rate (RR), and oxygen saturation from pulse oximetry $\left(\mathrm{SpO}_{2}\right)$. In a preliminary analysis of infants in a single NICU, we found that increased cross-correlation (or trending together, allowing for a lag) of $\mathrm{HR}$ and $\mathrm{SpO}_{2}$ performed well for preclinical detection of sepsis (12). Some of this increased cross-correlation likely represents changes in $\mathrm{HR}$ and $\mathrm{SpO}_{2}$ occurring in synchrony with pauses in breathing. In the current study, we expanded on this finding by analyzing vital signs from a large number of VLBW infants in two NICUs, both at baseline and surrounding two illnesses, late-onset septicemia (LOS) and NEC.

\section{METHODS}

Patient Population and Clinical Data Collection

We collected and stored all bedside monitor vital sign data on all patients admitted to the UVA NICU over a 64-mo period from 2009 to 2015 and to the Children's Hospital of New York NICU (Columbia University) over an 18-mo period from 2013 to 2015. All VLBW infants with data available were included in this study, which was 
Table 1. Vital signs and their cross-correlation over the entire NICU stay and in the $24 \mathrm{~h}$ period prior to LOS or NEC events (combined UVA and Columbia)

\begin{tabular}{|c|c|c|c|c|c|c|c|c|}
\hline Category & Variable & Measure & All mean (SD) & Event mean (SD) & ROC $_{\text {area }}{ }^{a}$ & $\boldsymbol{P}$ & VS + PMA ROC areab & $P$ \\
\hline \multirow[t]{7}{*}{ Vital signs } & Heart rate & Mean & $158(12)$ & $161(13)$ & 0.551 & 0.003 & 0.707 & 0.182 \\
\hline & Resp. Rate & & $51(11)$ & $50(12)$ & 0.540 & 0.174 & 0.701 & 0.243 \\
\hline & $\mathrm{SPO}_{2} \%$ & & $94(4)$ & $91(5)$ & 0.634 & 0.000 & 0.718 & 0.000 \\
\hline & Heart rate & Std. Dev. & $7(3)$ & $6(3)$ & 0.596 & 0.000 & 0.706 & 0.121 \\
\hline & Resp. Rate & & $17(5)$ & $17(6)$ & 0.530 & 0.054 & 0.701 & 0.365 \\
\hline & $\mathrm{SPO}_{2} \%$ & & $3(2)$ & $4(3)$ & 0.594 & 0.000 & 0.714 & 0.000 \\
\hline & $\mathrm{RR}-\mathrm{SPO}_{2}$ & & $0.23(0.12)$ & $0.25(0.13)$ & 0.527 & 0.091 & 0.699 & 0.137 \\
\hline \multirow[t]{4}{*}{ Demographics } & BWT & Grams & $920(288)$ & $817(261)$ & 0.605 & 0.000 & 0.715 & 0.000 \\
\hline & $\mathrm{GA}$ & Weeks & $26.8(2.6)$ & $25.9(2.5)$ & 0.604 & 0.000 & 0.707 & 0.035 \\
\hline & PMA & Weeks & $34.1(6.4)$ & $30.4(4.3)$ & 0.705 & 0.000 & NA & NA \\
\hline & Male & $\%$ & $54 \%$ & $55 \%$ & 0.503 & 0.860 & 0.705 & 0.796 \\
\hline
\end{tabular}

Bolded values are those with highest ROC area for vital signs and demographics.

${ }^{a} \mathrm{ROC}$ area comparing $0-24 \mathrm{~h}$ prior to LOS or NEC diagnosis to all data. ${ }^{\circ}$ Bivariate analysis,

BWT, birth weight; LOS, late-onset septicemia; Max., maximum; NEC, necrotizing enterocolitis; ROC, receiver operator characteristics curve.

approved by the Institutional Review Boards of both institutions with waiver of consent due to its purely observational nature.

Clinical data were abstracted from electronic medical records into a relational clinical database. Demographics included gestational age, birth weight, gender, and final outcome (death, discharge, or transfer). Cases of LOS and NEC were retrospectively identified from review of clinical databases. LOS was defined as signs of sepsis and a positive blood culture at 3 or more days of age and at least $5 \mathrm{~d}$ of treatment with antibiotics. Subsequent episodes of LOS or NEC were included if they occurred more than $7 \mathrm{~d}$ after the previous episode. NEC was defined as clinical and radiographic signs of NEC and a full course of therapy (bowel rest and antibiotics). We excluded cases of focal intestinal perforation without NEC as identified by the attending neonatologist and pediatric surgeon, based on clinical and, when available, surgical findings. Cases in which infants were transferred from an outside hospital with LOS or NEC were excluded since baseline "well" data were not available for comparison.

Data collected related to LOS or NEC episodes included chronologic and postmenstrual age at the time of the blood culture or abdominal radiograph establishing the diagnosis, blood culture results, and whether on ventilatory support at the time of diagnosis.

\section{Vital Sign Analysis}

Bedside monitor data were collected using a BedMaster central network server (Excel Medical, Jupiter, FL). RR derived from the chest impedance signal, HR from the ECG signal, and $\mathrm{SpO}_{2}$ from the pulse oximeter were collected every $2 \mathrm{~s}$. During the time period of study at both institutions, pulse oximeters were set to the default $\mathrm{SpO}_{2}$ averaging setting ( $8 \mathrm{~s}$ ). Mean, $\mathrm{SD}$, and cross-correlation of $\mathrm{HR}, \mathrm{RR}$, and $\mathrm{SpO}_{2}$ were calculated in $10 \mathrm{~min}$ windows and then averaged for each hour for analysis throughout the NICU stay and then specifically in the 2-d period before and after diagnosis of LOS and NEC.

\section{Quantifying Cross-Correlation Between Vital Signs}

The maximum cross-correlation between two vital sign signals was measured over 10-min windows by first standardizing each signal (subtracting mean and dividing by standard deviation) and then using the Matlab function XCORR, with a lag time of -30 to $+30 \mathrm{~s}$. A high value of this statistic (approaching 1 ) indicates the two signals are in positive synchrony (i.e., they go up and down together) with a possible lag or time difference of up to $30 \mathrm{~s}$. We also calculated the minimum cross-correlation value reflecting signals going in opposite directions; these negative synchrony values are not reported because they were not associated with adverse events.

\section{Heart Rate Characteristics Index Analysis}

At UVA, a heart rate characteristics (HRC) index monitor has been in use since 2003 (HeRO monitor, Medical Predictive Science Corporation, Charlottesville, VA). The monitor was developed as an early warning system for sepsis, and the HRC index incorporates three measures of abnormal HR characteristics that occur in some neonates with sepsis: low HR variability, sample asymmetry (more decelerations, fewer accelerations), and low sample entropy. The HRC index is displayed at UVA and not at Columbia, and we therefore compared vital sign metrics examined in this study with the HRC index in UVA patients only.

\section{Statistics and Modeling}

Summary statistics and logistic regression were used to describe and compare vital signs collected from infants at UVA and Columbia. Univariate logistic regression analyses were performed to determine whether there was a significant change in each vital sign metric in the $24 \mathrm{~h}$ period before diagnosis of LOS or NEC, compared to normative data from all VLBW infants at all times. Demographic variables potentially associated with LOS and NEC (gestational age, birth weight, gender, and postmenstrual age) were also analyzed. Bivariate analyses incorporating postmenstrual age and each vital sign metric were performed. Multivariate logistic regression models were developed using data from each site separately as the training set in an iterative process with external validation on data from the other site.

For summary statistics, mean (SD) is shown unless otherwise indicated. For associations between vital signs and illnesses, Wald Chi-square and $P$ values are reported, and for modeling, area under receiver operator characteristics curve (ROC AUC) and 95\% confidence intervals (CI) are reported. Net reclassification improvement was also used to compare the performance of the new vital sign models with the HRC index. Net reclassification improvement is a measure of the fraction of cases that are correctly reclassified by a new risk assessment tool compared to an established tool (13).

Analyses were performed in MATLAB (MathWorks, Natick MA).

\section{RESULTS}

\section{Patient Population and LOS and NEC Cases}

Of 1,125 VLBW infants admitted to the two NICUs during the study period, vital sign data were available for 1,065 (757 in 64 mo at The University of Virginia, UVA, and 308 in 18 mo at Columbia University). Gestational age and birth weight 
a

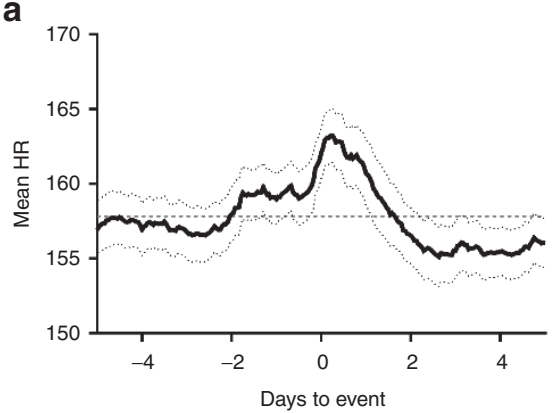

d

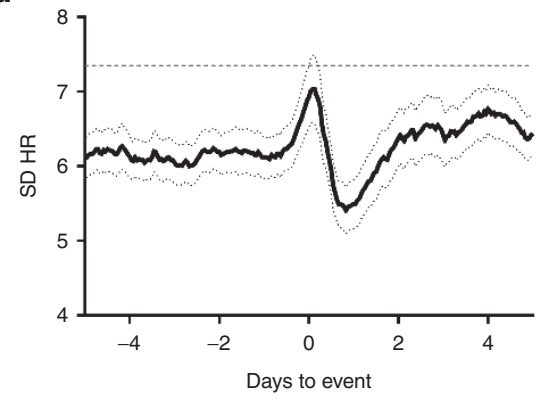

g

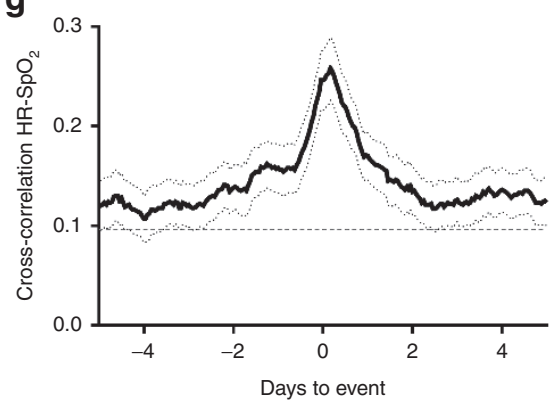

b

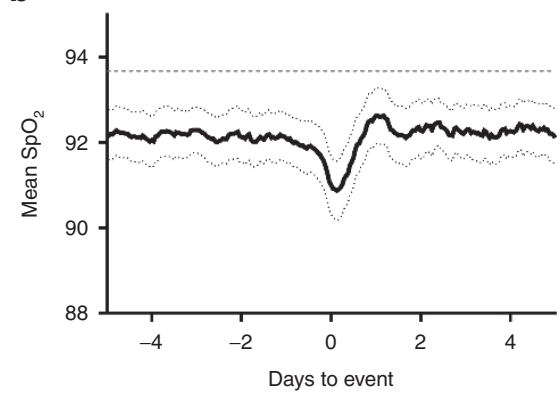

e

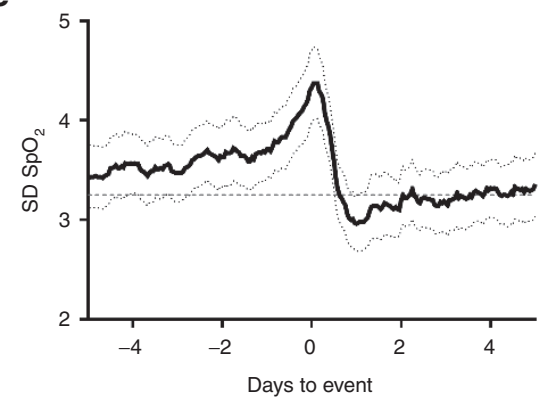

h

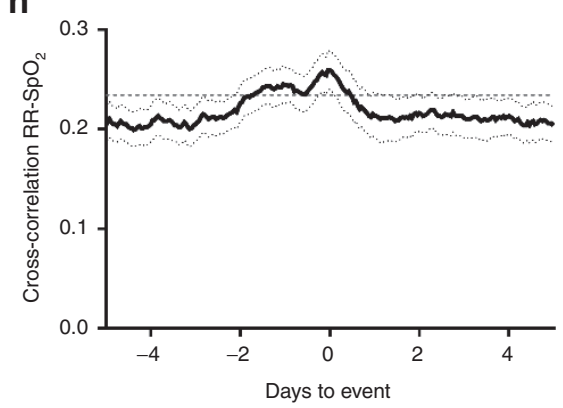

c

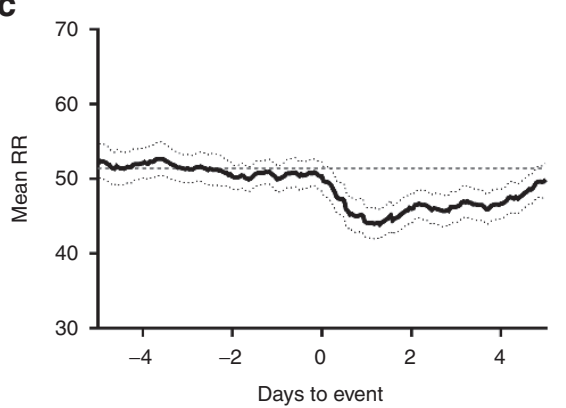

f

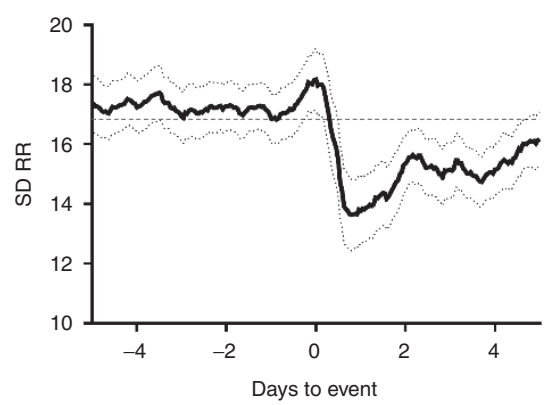

i

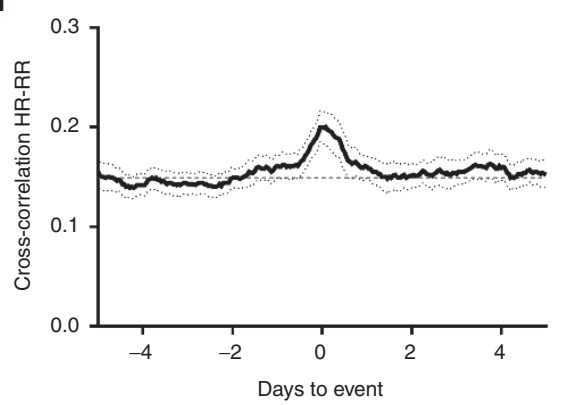

Figure 1. Vital signs and their cross-correlation around septicemia and necrotizing enterocolitis (NEC) events. In 186 cases of late-onset septicemia or NEC at UVA and Columbia, mean (panels a-c), SD (panels $\mathbf{d}-\mathbf{f}$ ) and cross-correlation (panels $\mathbf{g}-\mathbf{i}$ ) of heart rate (HR), oxygen saturation (SpO ${ }_{2}$ ) and respiratory rate (RR), are shown $5 \mathrm{~d}$ before and after diagnosis. Mean (solid line) and $95 \%$ confidence interval (dotted line) are shown. The horizontal dashed line represents the value for all very-low-birth-weight infants at all times.

were similar at the two institutions (UVA: $27.6 \pm 2.9 \mathrm{wk}$ and $1,003 \pm 297 \mathrm{~g}$; Columbia: $28.5 \pm 3.2 \mathrm{wk}$ and $1,030 \pm 313 \mathrm{~g}$ ). The total number of infant-years' vital sign data available for analysis was 95 and 36 for UVA and Columbia infants.

Among the 1,065 infants, there were 123 cases of LOS and 63 cases of NEC with vital sign data available around the time of illness. Mean gestational age and birth weight of infants with LOS or NEC were $25.9 \mathrm{wk}$ and $817 \mathrm{~g}$, significantly lower than infants without these illnesses (Table 1). Organism distribution for the LOS cases was 88 (72\%) Gram-positive, 34 (28\%) Gram-negative or multiple organisms, and one Candida species. Demographics and organisms for the LOS and NEC cases were similar at the two institutions. Infants with LOS were more likely to be on mechanical ventilation at the time of diagnosis $(65 / 123,53 \%)$ compared to infants with NEC $(13 / 65,21 \%)$, and infants at UVA were more likely to be on mechanical ventilation at the time of LOS or NEC $(64 / 121,53 \%)$ compared to infants at Columbia $(14 / 65,22 \%)$.

\section{Vital Sign and Model Analysis}

Mean, SD, and cross-correlation of $\mathrm{HR}, \mathrm{RR}$, and $\mathrm{SpO}_{2}$ were analyzed during all times data were available. Total number of hours of data analyzed was 1.15 million (130.9 infant-years), and the breakdown by institution was UVA, 0.84M (95.4 infant-years) and Columbia, $0.31 \mathrm{M}$ (35.5 infant-years).

Table 1 summarizes demographic variables and mean vital signs for the entire NICU stay for all 1,065 infants, and vital signs in the 24-h period before diagnosis of either LOS or NEC. Mean postmenstrual age (PMA) at the time of illness was $30.4 \mathrm{wk}$. In univariate analysis, the best predictor of LOS or NEC being diagnosed within $24 \mathrm{~h}$ was PMA. In multivariate analysis adjusting for PMA, cross-correlation of $\mathrm{HR}-\mathrm{SpO}_{2}$ had the highest ROC area for LOS or NEC $(0.733, P<0.001)$.

Figure 1 shows means of each vital sign-related parameter in the 5-d period before and after LOS or NEC diagnosis compared to the population mean for all infants for the entire NICU stay (horizontal gray line). Generally, mean HR increased slightly and mean $\mathrm{SpO}_{2}$ decreased slightly around 


\section{Articles $\mid$ Fairchild et al.}

Table 2. Site-specific vital sign and model performance for late-onset septicemia and necrotizing enterocolitis detection

\begin{tabular}{|c|c|c|c|c|c|c|}
\hline \multirow[b]{2}{*}{ Vital sign ${ }^{a}$} & \multicolumn{2}{|c|}{ UVA } & \multicolumn{2}{|c|}{ CU } & \multicolumn{2}{|c|}{ Combined } \\
\hline & Chi-squared & $P$ & Chi-squared & $P$ & Chi-squared & $P$ \\
\hline Mean heart rate & 7.201 & 0.0073 & 0.881 & 0.3479 & 3.198 & 0.0737 \\
\hline Mean Resp Rate & 6.104 & 0.0135 & 15.242 & 0.0001 & 18.439 & 0.0000 \\
\hline Mean $\mathrm{SPO}_{2} \%$ & 20.826 & 0.0000 & 0.547 & 0.4596 & 24.974 & 0.0000 \\
\hline SDHR & 17.971 & 0.0000 & 30.885 & 0.0000 & 38.675 & 0.0000 \\
\hline SD RR & 4.491 & 0.0341 & 11.830 & 0.0006 & 13.615 & 0.0002 \\
\hline $\mathrm{SDSPO}_{2}$ & 0.475 & 0.4906 & 8.159 & 0.0043 & 0.199 & 0.6558 \\
\hline XC HR-RR & 7.404 & 0.0065 & 3.420 & 0.0644 & 9.023 & 0.0027 \\
\hline $\mathrm{XCHR}-\mathrm{SPO}_{2}$ & 36.487 & 0.0000 & 36.530 & 0.0000 & 73.957 & 0.0000 \\
\hline XC RR-SPO & 0.650 & 0.4203 & 8.522 & 0.0035 & 0.946 & 0.3307 \\
\hline Model & ROC area & $95 \% \mathrm{Cl}$ & ROC area & $95 \% \mathrm{Cl}$ & ROC area & $95 \% \mathrm{Cl}$ \\
\hline 9-variable model & 0.711 & $0.674-0.734$ & 0.770 & $0.727-0.812$ & 0.721 & $0.691-0.743$ \\
\hline 3-variable model ${ }^{b}$ & 0.695 & $0.654-0.721$ & 0.745 & $0.710-0.786$ & 0.710 & $0.679-0.728$ \\
\hline $\mathrm{XC} \mathrm{HR}-\mathrm{SpO}_{2}$ & 0.617 & $0.577-0.651$ & 0.701 & $0.654-0.752$ & 0.644 & $0.618-0.673$ \\
\hline
\end{tabular}

Bolded chi-squared values indicate those included in the 3-variable model.

${ }^{a}$ Contribution to the 9-variable model. ' ${ }^{\mathrm{M}} \mathrm{Mean} \mathrm{SpO}_{2}, \mathrm{SDHR}$, and $\mathrm{XCHR-SpO}$ XC cross-correlation.

$\mathrm{Cl}$, confidence interval; $\mathrm{ROC}$, receiver operator characteristics curve.

the time of diagnosis (panels a, b). Mean RR did not increase prior to diagnosis but its standard deviation did (panels $c, f$ ), which may reflect infants having more fluctuations between tachypnea and apnea. Vital sign parameters changed after diagnosis, possibly related to therapeutic interventions such as increased respiratory support. Of note, changes in vital sign measures in the day prior to illness differed between centers. For example, there was a small but statistically significant decrease in mean $\mathrm{SpO}_{2}$ and increase in mean $\mathrm{HR}$ at UVA and not at Columbia, whereas $\mathrm{SD}$ of $\mathrm{SpO}_{2}$ changed before illness at Columbia and not at UVA. Importantly, though, the crosscorrelation of $\mathrm{HR}-\mathrm{SpO}_{2}$, which measures cotrending of the two vital signs rather than their absolute values, was the best single predictor of illness at both centers (panel g).

Table 2 shows center-specific vital sign model ability to discriminate infants with impending LOS or NEC. For modeling, the UVA training set consisted of 825,493 hourly measurements with an event rate of 0.0032 and the Columbia training set consisted of 235,458 hourly measurements with an event rate of 0.0035 . The contribution of each vital sign to the 9-variable model is represented as a Chi-squared value and corresponding coefficient $P$ value. Performance and CI of the 9-variable model and a model using the three best independent predictors (mean $\mathrm{SpO}_{2}, \mathrm{SD} \mathrm{HR}$, and cross-correlation $\mathrm{HR}-\mathrm{SpO}_{2}$ ) is shown. The table shows results of training and testing the model at each site separately and combined. We also performed external cross-validation. The UVA 3-variable model had an AUC of 0.754 when tested on Columbia data and the Columbia model had AUC 0.680 tested on UVA data. The 9-variable model had slightly less external validated performance with corresponding AUCs of 0.727 and 0.674 .

\section{Cross-Correlation of HR-SpO 2 Prior to LOS and NEC Diagnosis}

Figure 2a shows that the distribution of cross-correlation of $\mathrm{HR}-\mathrm{SpO}_{2}$ measurements was skewed toward higher values in the $24 \mathrm{~h}$ period prior to LOS or NEC diagnosis compared to the values of all infants at all times. The rise in cross-correlation $\mathrm{HR}-\mathrm{SpO}_{2}$ was greater in cases of NEC than LOS and continued to rise after diagnosis (Figure $2 \mathbf{b}$ ). The effect was seen in infants at both institutions (Figure 2c) and in infants on or off mechanical ventilation at the time of diagnosis (Figure $2 \mathrm{~d}$ ). Combining LOS and NEC cases and both institutions, mean cross-correlation of $\mathrm{HR}-\mathrm{SpO}_{2}$ increased from 0.15 in the 24-48 h period prior to diagnosis to 0.21 from $0-24 \mathrm{~h}$ prior $(P<0.001)$.

\section{Examples of High Cross-Correlation of $\mathrm{HR}-\mathrm{SpO}_{2}$ in Relation to Respiratory Rate}

In $33 \%$ of the LOS or NEC events (61/186), at least one of the hours in the day prior to the event had an extremely high cross-correlation $>0.6$ corresponding to the tail of the distribution in Figure 2a. We speculated that, in some cases, this finding represents apnea or periodic breathing with associated decline in $\mathrm{HR}$ and $\mathrm{SpO}_{2}$, and we reviewed respiratory rate, $\mathrm{HR}$, and $\mathrm{SpO}_{2}$ patterns the day prior to illness diagnosis in all cases with very high cross-correlation. Representative examples are shown in Figure 3. In some cases, there was clearly central apnea associated with decline in $\mathrm{HR}$ and $\mathrm{SpO}_{2}$ deep enough to be considered "bradycardia/desaturation" by standard definitions (Figure 3a) (14). In others, the concurrent fall and rise in $\mathrm{HR}, \mathrm{SpO}_{2}$, and $\mathrm{RR}$ were of lower magnitude and duration but higher frequency, suggestive of periodic breathing with entrainment of $\mathrm{HR}$ and $\mathrm{SpO}_{2}$ (Figure 3b).

\section{Vital Sign Metrics Compared to and Added to an Established Heart Rate Characteristics Index}

An HRC index (HeRO) monitor was in use at UVA during the period of study, and scores were available for 620,978 of the hourly measurements used in the UVA vital sign training set. For this subset, the additional value of the parsimonious 3-parameter 

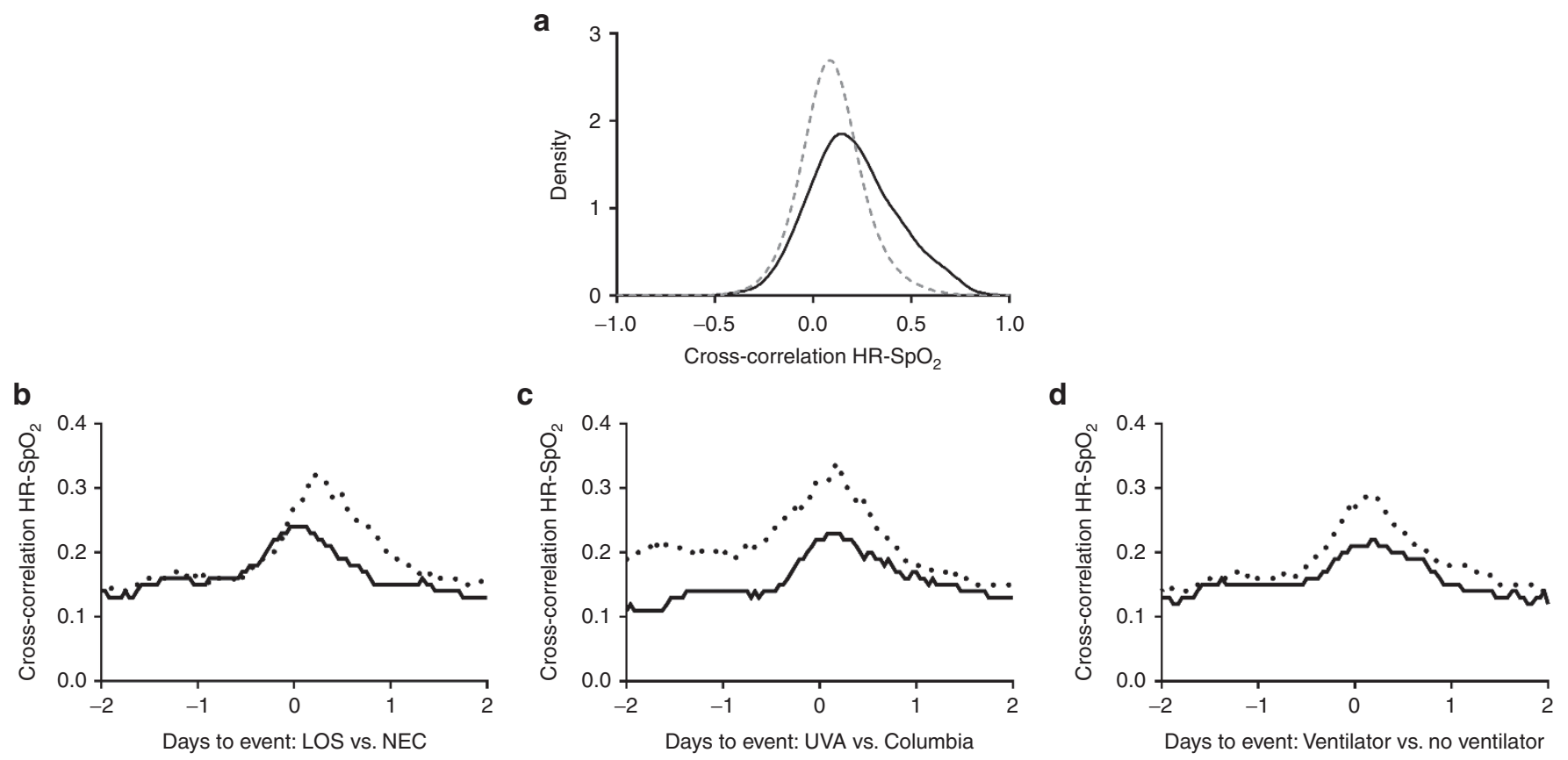

Figure 2. Cross-correlation of $\mathrm{HR}-\mathrm{SpO} 2$ in septicemia and necrotizing enterocolitis (NEC). Vital sign data were analyzed for cross-correlation of $\mathrm{HR}-\mathrm{SpO}{ }_{2}$ for 1,065 very-low-birth-weight (VLBW) infants at all times and around the time of 123 cases of late-onset septicemia (LOS) and 63 cases of NEC. Panel a) For each cross-correlation value, the density of hourly measurements for all 1,065 VLBW infants at all times during the NICU stay is shown by the grey dashed line and the number of measurements in the $24 \mathrm{~h}$ period prior to illness diagnosis by the black line. (Panel $\mathbf{b}-\mathbf{d}$ ) Mean cross-correlation of $\mathrm{HR}-\mathrm{SpO}_{2} 2 \mathrm{~d}$ prior to and following diagnosis of illness. Increased cross-correlation occurred in both illnesses (panel $\mathbf{b}$, LOS solid line, versus NEC dotted line), in both institutions (panel c, UVA solid line, versus Columbia dotted line), and in infants on or off mechanical ventilation (panel d, on ventilator solid line, off ventilator dotted line).

vital sign model to the HRC index were evaluated using logistic regression models and the net reclassification improvement statistic (13). The AUC on this subset of data for the vital sign model and HRC index alone were 0.684 and 0.707 respectively. Combining the 3-variable model with the HRC index increased AUC by 0.021 to 0.728 ( $95 \%$ CI: $0.010,0.047$; Wald chi-square $=22.6 ; P=0.00001)$. The Net Reclassification Improvement for the vital sign model was also highly significant with a value of $0.205(0.113,0.328)$. The cross-correlation $\mathrm{HR}_{-} \mathrm{SPO}_{2}$ by itself also added significantly to the HRC index (Wald chi-square $=9.14, P$ $=0.01$ ) with combined AUC of 0.715 . This analysis demonstrates the potential of additional vital sign analyses to improve the sensitivity over the established heart rate characteristics index monitor for early detection of LOS and NEC.

\section{DISCUSSION}

We previously developed a monitor displaying a heart rate characteristics index as an early warning system for sepsis, and in the current study we analyzed not only HR, but also respiratory rate and $\mathrm{SpO}_{2}$. We further expanded the study by analyzing data from a large number of VLBW infants at two institutions and in two illnesses. The major finding is that, while the value of individual vital signs for detection of LOS and NEC differed across institutions, an increase in cross-correlation of $\mathrm{HR}-\mathrm{SpO}_{2}$ performed well in both units and added to the HRC index for early detection of illness.

High cross-correlation of HR-SpO $\mathrm{P}_{2}$ may reflect apnea in infants who are not on mechanical ventilation. An acute increase in central apnea is one of the most common signs of late-onset septicemia in preterm infants in the NICU (15), and apnea is often accompanied by both bradycardia and oxygen desaturation. Periodic breathing, alternating brief apneic pauses and breaths, is normal in neonates and sometimes associated with decline in $\mathrm{HR}$ and $\mathrm{SpO}_{2}$. Preclinical studies indicate that cytokines and prostaglandins released as part of the systemic inflammatory response are responsible for emergence of immature breathing patterns during illness $(16,17)$, and we have previously reported that some preterm infants exhibit an acute increase in periodic breathing or in central"ABDs" (apnea with both bradycardia and desaturation) in the day before they are diagnosed with LOS or NEC $(10,11)$. Quantitation of central apnea requires storage and analysis of large data files of chest impedance waveforms which is difficult to implement broadly, and in the current study we sought simpler measures using vital signs and their interactions. On reviewing examples of very high cross-correlation of $\mathrm{HR}-\mathrm{SpO}_{2}$, we found that many were clearly associated with decline in breathing rate consistent with central apnea (Figure 3a) and periodic breathing (Figure 3b), and further work is needed to substantiate this association. Interestingly, some infants on mechanical ventilation also had high cross-correlation of $\mathrm{HR}_{-} \mathrm{SpO}_{2}$ at the time of LOS or NEC diagnosis, indicating that there is more at play in the pathophysiology than central apnea. We speculate that some of this may reflect autonomic nervous system activation or dysfunction, or altered vasoreactivity as part of a systemic inflammatory response. 


\section{Articles $\mid$ Fairchildetal.}
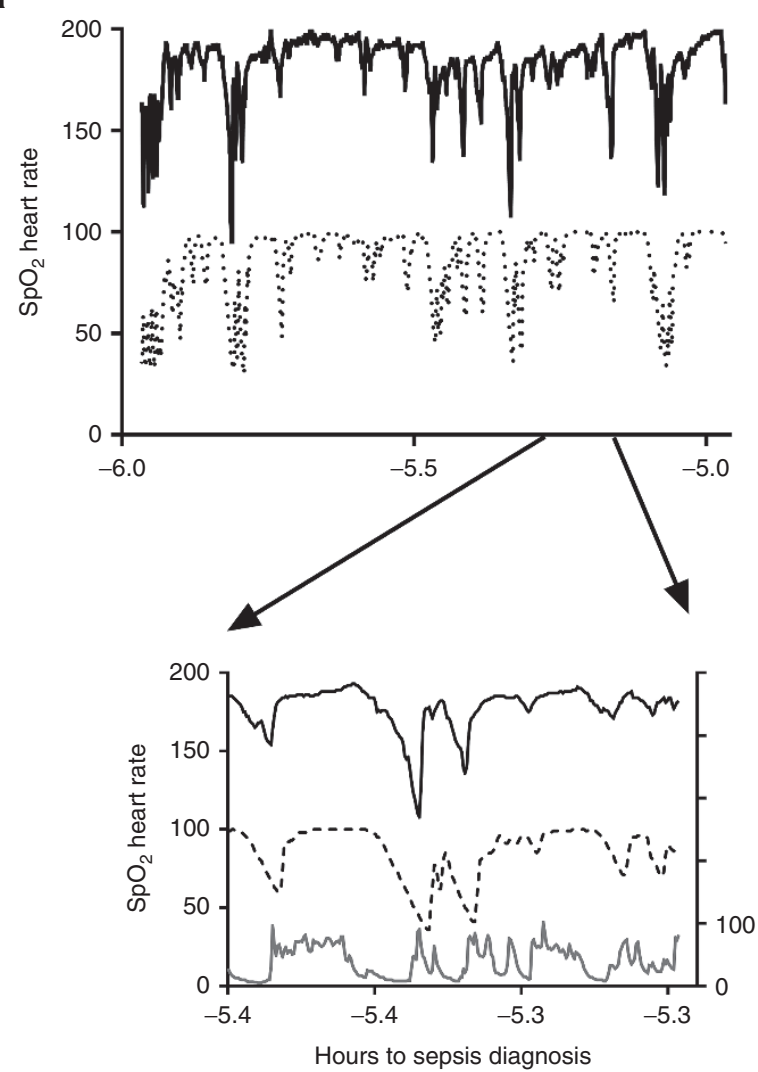

b

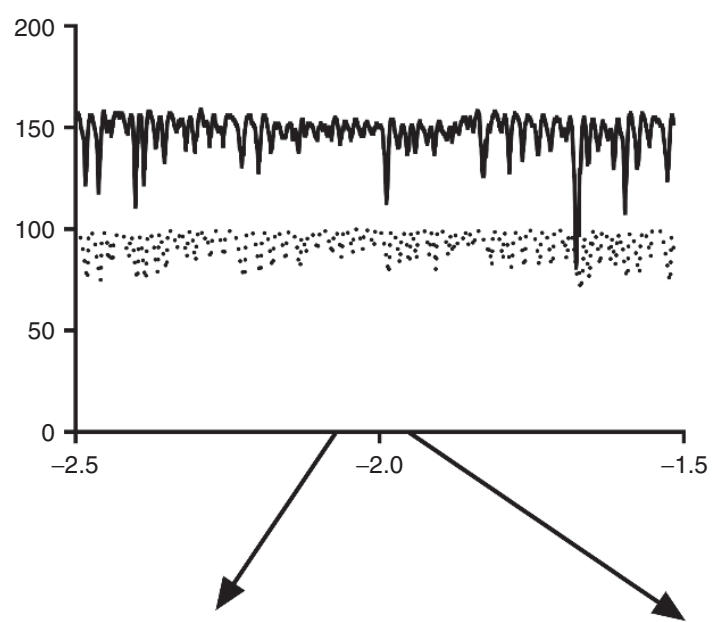

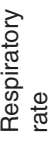

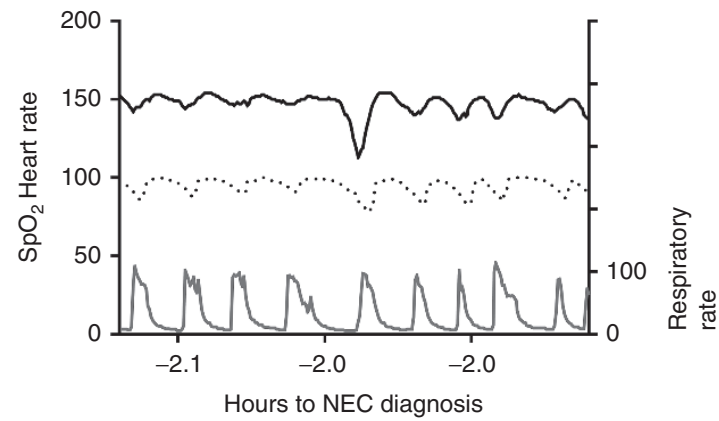

Figure 3. Examples of high cross-correlation of $\mathrm{HR}_{-} \mathrm{SpO}_{2}$ and associated respiratory rate patterns prior to septicemia and necrotizing enterocolitis (NEC). Representative $1 \mathrm{~h}$ tracing of $\mathrm{HR}$ and $\mathrm{SpO}_{2}$ (top), and 10 min tracings of $\mathrm{HR}, \mathrm{SpO}_{2}$, and respiratory rate (insets at bottom) are shown for two infants in the day prior to diagnosis of illness. Panel a) UVA infant $5 \mathrm{~h}$ prior to diagnosis of sepsis, when cross-correlation of HR-SpO, was 0.844 . There are frequent $\mathrm{HR}$ decelerations (solid black line) and concurrent decline in $\mathrm{SpO}_{2}$ (dotted black line), preceded by decline in respiratory rate (solid gray line in bottom inset, note right y-axis for respiratory rate). (Panel b) Columbia infant $1.5 \mathrm{~h}$ prior to diagnosis of NEC, when cross-correlation of HR-SpO ${ }_{2}$ was 0.81 . There are repetitive, regular declines in $\mathrm{HR}$ and $\mathrm{SpO}_{2}$ associated with decline in respiratory rate.

In order for predictive models to be widely applicable, it is important that they are developed and tested at different institutions. We found small but statistically significant differences in individual vital signs of infants at UVA and Columbia, most notably, mean and $\mathrm{SD}$ of $\mathrm{SpO}_{2}$ and their contribution to the predictive models (Table 2). This may reflect differences in clinical management strategies of lung disease or apnea, and may account for the more robust performance of vital sign interactions such as cross-correlation of $\mathrm{HR}-\mathrm{SpO}_{2}$ across institutions, since this measure reflects cotrending rather than absolute values. Another consideration for the predictive models we report here is that they compare vital sign patterns before illness with those of all VLBW infants at all times during the NICU stay. Since vital signs and occurrence of apnea and periodic breathing change with advancing gestational and postmenstrual age, an important future direction is to incorporate demographic and clinical variables into the algorithm. Furthermore, since there may be significant interindividual variation in vital signs and apnea, displaying an acute change compared to an infant's baseline might be the best approach.

Any new diagnostic or predictive test such as cross-correlation of $\mathrm{HR}-\mathrm{SpO}_{2}$ should be compared to existing modalities. The HRC index was previously developed by our group as an early warning system for sepsis and is displayed in the UVA NICU. Whether display of the HRC index led to treatment of the UVA infants at an earlier phase of illness is unknown since we cannot pinpoint the exact time an infant develops bacteremia or NEC. We did find that infants at Columbia had a higher cross-correlation of $\mathrm{HR}-\mathrm{SpO}_{2}$ in the 2-d period prior to diagnosis ( 0.2 vs. 0.1 , Figure 2 c) which could reflect more severe or longer-standing illness. Alternatively, this might simply reflect the fact that more UVA infants were on mechanical ventilation at the time of diagnosis and therefore did not have apnea or periodic breathing. Of note, we found that there was additive value in combining the HRC index and the cross-correlation of $\mathrm{HR}-\mathrm{SpO}_{2}$ or the 3-vital sign model for early detection of LOS and NEC.

More work is required to determine whether displaying a score representing multiple vital signs will improve outcomes of infants in the NICU. An important aspect of this work is to determine whether to identify a threshold "alarm" value with acceptable sensitivity, false-positive rate, and other diagnostic utility measures. A major problem with this approach is that infants in the early stages of illness who have worsening vital sign patterns compared to their own baseline but who fall below the threshold will be missed. Another problem is that some infants at high risk 
for sepsis or NEC may have chronically abnormal vital sign patterns (above the threshold). An alternative monitoring strategy, favored by our group and used for the HRC index, is to display in real-time a vital sign-based risk score, so that trends over time for individual infants can be used in decisions about testing and treatment for acute and potentially catastrophic illnesses $(4,18)$.

\section{Conclusion}

Sepsis and NEC continue to contribute a great deal to morbidity and mortality of preterm infants (19). Detection and treatment in the early phase of illness, before overt clinical deterioration, is likely to improve outcomes but is difficult due to the subtlety of the early physiologic changes. Analysis of multiple vital signs and their interactions can assist in preclinical detection in some cases, and translating these metrics to real-time bedside displays and testing their impact on outcomes in randomized clinical trials is an essential next step.

\section{STATEMENT OF FINANCIAL SUPPORT}

This study was supported by National Institutes of Health (Bethesda, MD) grant HD072071.

Disclosures: None.

\section{REFERENCES}

1. Lake DE, Fairchild KD, Moorman JR. Complex signals bioinformatics: evaluation of heart rate characteristics monitoring as a novel risk marker for neonatal sepsis. J Clin Monit Comput 2014;28:329-39.

2. Mithal LB, Yogev R, Palac H, Gur I, Mestan KK. Computerized vital signs analysis and late onset infections in extremely low gestational age infants. J Perinat Med 2016;44:491-7.

3. Bravi A, Green G, Longtin A, Seely AJ. Monitoring and identification of sepsis development through a composite measure of heart rate variability. PLoS One 2012;7:e45666.

4. Moorman JR, Carlo WA, Kattwinkel J, et al. Mortality reduction by heart rate characteristic monitoring in very low birth weight neonates: a randomized trial. J Pediatr 2011;159:900-6.e1.

5. Griffin MP, O'Shea TM, Bissonette EA, Harrell FE Jr, Lake DE, Moorman JR. Abnormal heart rate characteristics preceding neonatal sepsis and sepsis-like illness. Pediatr Res 2003;53:920-6.
6. Griffin MP, Lake DE, Bissonette EA, Harrell FE Jr, O’Shea TM, Moorman JR. Heart rate characteristics: novel physiomarkers to predict neonatal infection and death. Pediatrics 2005;116:1070-4.

7. Fairchild KD, Schelonka RL, Kaufman DA, et al. Septicemia mortality reduction in neonates in a heart rate characteristics monitoring trial. Pediatr Res 2013;74:570-5.

8. Fairchild KD. Predictive monitoring for early detection of sepsis in neonatal ICU patients. Curr Opin Pediatr 2013;25:172-9.

9. Sullivan BA, Fairchild KD. Predictive monitoring for sepsis and necrotizing enterocolitis to prevent shock. Semin Fetal Neonatal Med 2015;20: 255-61.

10. Patel M, Mohr M, Lake D, et al. Clinical associations with immature breathing in preterm infants: part 2-periodic breathing. Pediatr Res 2016;80: 28-34.

11. Fairchild $\mathrm{K}$, Mohr M, Paget-Brown A, et al. Clinical associations of immature breathing in preterm infants: part 1-central apnea. Pediatr Res 2016;80:21-7.

12. Moss TJ, Lake DE, Calland JF, et al. Signatures of subacute potentially catastrophic illness in the ICU: model development and validation. Crit Care Med 2016;44:1639-48.

13. Leening MJ, Vedder MM, Witteman JC, Pencina MJ, Steyerberg EW. Net reclassification improvement: computation, interpretation, and controversies: a literature review and clinician's guide. Ann Intern Med 2014;160:122-31.

14. Finer NN, Higgins R, Kattwinkel J, Martin RJ. Summary proceedings from the apnea-of-prematurity group. Pediatrics 2006;117:S47-51.

15. Das A, Shukla S, Rahman N, Gunzler D, Abughali N. Clinical indicators of late-onset sepsis workup in very low-birth-weight infants in the neonatal intensive care unit. Am J Perinatol 2016;33:856-60.

16. Hofstetter AO, Saha S, Siljehav V, Jakobsson PJ, Herlenius E. The induced prostaglandin E2 pathway is a key regulator of the respiratory response to infection and hypoxia in neonates. Proc Natl Acad Sci USA 2007;104: 9894-9.

17. Balan KV, Kc P, Hoxha Z, Mayer CA, Wilson CG, Martin RJ. Vagal afferents modulate cytokine-mediated respiratory control at the neonatal medulla oblongata. Respir Physiol Neurobiol 2011;178:458-64.

18. Pepe MS, Feng Z, Huang $Y$, et al. Integrating the predictiveness of a marker with its performance as a classifier. Am J Epidemiol 2008;167:362-8.

19. Stoll BJ, Hansen NI, Bell EF, et al.; Eunice Kennedy Shriver National Institute of Child Health and Human Development Neonatal Research Network. Trends in Care Practices, Morbidity, and Mortality of Extremely Preterm Neonates, 1993-2012. JAMA 2015;314:1039-51. 